\title{
Ferromagnetic Glass-Coated Micro-and Nanowires for Medical Application
}

\author{
SA Baranov ${ }^{1,2 *}$ \\ ${ }^{1}$ Institute of Applied Physics, Academiei str. 5, Chisinau, MD 2028 Republic of Moldova, Russia \\ ${ }^{2}$ Shevchenko Pridnestrov'e State University, str. 25 Oktyabrya 128, Tiraspol, Republic of Moldova, Russia
}

*Corresponding author: SA Baranov, Institute of Applied Physics, Academiei str. 5, Chisinau, MD 2028, Shevchenko Pridnestrov'e

State University, str. 25 Oktyabrya 128, Tiraspol, Republic of Moldova, Russia

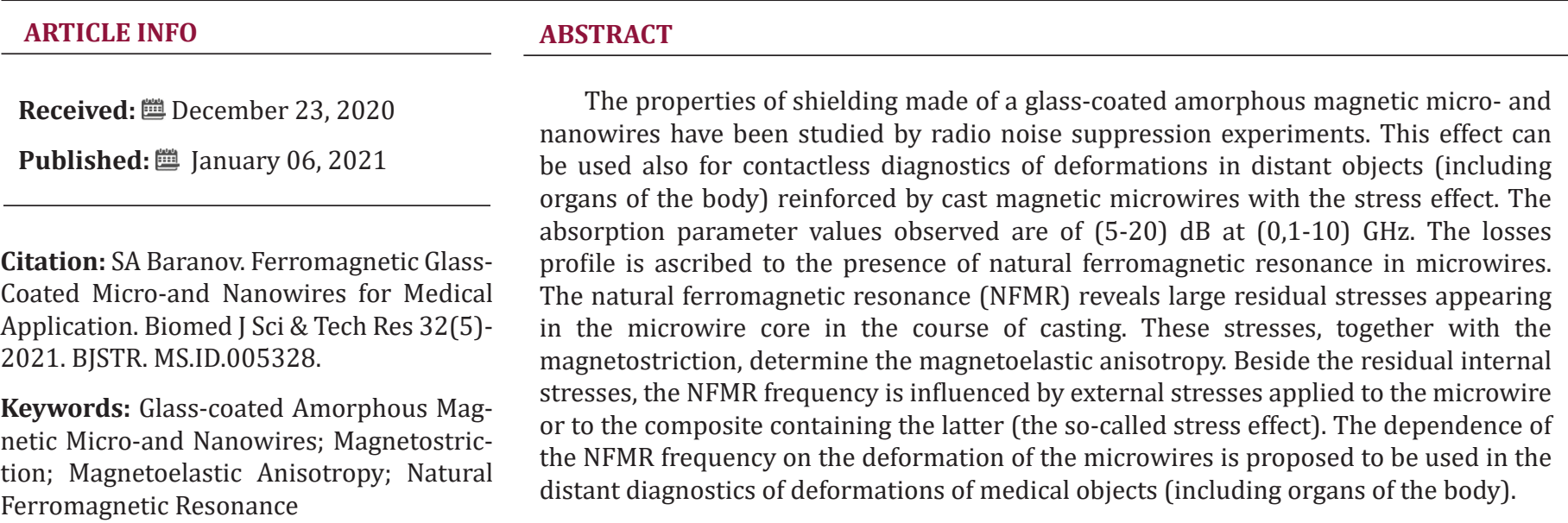

\section{Introduction}

The increasingly miniaturized devices at high frequencies lead to noise in medical apparats. This fact has strongly promoted the development for noise suppression purposes [1-3]. Application magnetic glass-coated cast amorphous micro and nanowires (GCAMNWs) is considered also in articles [1-4]. The properties of the magnetic glass-coated cast amorphous micro and nanowires studies in many publications by various research groups [1-16]. As this research has evolved, the need to control the magnetic anisotropy in components came into attention since the field absorption is related to natural ferromagnetic resonance (NFR) [510]. That glass-coated amorphous magnetic micro-and nanowires (GCAMNWs) possess very interesting high frequency properties due to the strong magnetoelastic anisotropy which is induced in the fabrication process [5]. By defining strictly, the geometric characteristics and the alloy composition the medical norms of electromagnetic field can be accurately controlled. Furthermore, the high production rate and high permeability feature of GCAMNWs without any rigid substrates are advantageous for use as noise absorbers of electromagnetic field. In this report, amorphous glasscoated microwire arrays in a sheet configuration are proposed for use as absorbers. High frequency characteristics of thin amorphous microwires will be presented and evaluated as radio noise absorbers and medical protection radio absorption shielding.

During the manufacturing, the GCAMNWs using the UlitovskyTaylor method the soften glass capillary is filled with a melted metal alloy with a further quenching [5]. The residual stresses, arising in metal core of GCAMNWs by during manufacturing, determine magnetic behavior of such GCAMNWs [5]. The metal is frozen into very thin GCAMNWs. Thus, a strong quenching stress (for example, $s \sim 10^{9} \mathrm{~Pa}$ in ultrathin GCAMNWs) is introduced in the GCAMNWs [4-12]. The phenomenon of natural ferromagnetic resonance (NFMR) in CGCMMWs [5-14] is extremely interesting from the viewpoint of using it for non-contact diagnostics of distant objects (or organs of the body). The diagnostics become possible due to 
the stress effect on the NFMR, that is, the shift of the resonance frequency as a result of a deformation of the object (or organs of the body). Such a frequency shift can be measured by irradiating the object with microwaves emitted by radar at frequencies near the NFMR and detecting the reflected signal, thus revealing a deviation of the resonance frequency from the original value. The glass coating of the cast GCAMNWs induces strong mechanical stresses in the kernel [4-12]. In cylindrical coordinates, the residual tension is characterized by the axial, $\sigma_{z}$, radial, $\sigma_{r}$, and tangential, $\sigma_{\varphi}$, components. The value of these stresses depends on the ratio of the radius, $\mathrm{Rm}$, of the metallic kernel to the total microwire radius, Rc:

$$
x=\left(\frac{R_{c}}{R_{m}}\right)^{2}-1,
$$

Using the cylinder-shell model, we then obtain a formula for stresses in the metallic kernel of the cast GCAMNWs:

$$
\begin{aligned}
& \sigma_{r}=\sigma_{\phi} \equiv P_{v}, \\
& P_{v}=\varepsilon E_{1} \frac{k x}{[k(1-2 v)+1] x+2(1-v),}
\end{aligned}
$$

Where

$$
\varepsilon E_{1}=\sigma_{0} \sim 2 G P a,
$$

is the maximum stress in the metallic kernel; $\varepsilon$ is the difference between the thermal expansion of the metallic kernel and that of the glass shell with the expansion coefficients $\alpha_{1}$ and $\alpha_{2}: \varepsilon=(\alpha 1-$ $\alpha 2)\left(T^{*}-T\right) ; \quad E 1$ is the Young modulus of the metallic kernel, $T^{*}$ is the solidification temperature of the composite in the metal /glass contact region $\left(\mathrm{T}^{*} \sim(800 \ldots 1200) \mathrm{K}\right), \mathrm{T}$ is the room temperature; $\mathrm{k}$ is the ratio between Young's moduli of the glass and the metal ; $v$ is the Poisson ratio. Let us consider the case where all the Poisson ratios are $v=1 / 3$ in order to obtain

$$
P=\varepsilon E_{1} \frac{k x}{(k / 3+1) x+4 / 3}
$$

$$
\sigma_{z}=P \frac{(K+1) x+2}{K x+1}
$$

With additional longitudinal strain, which occurs when the microwire is embedded in a solid matrix that itself deforms under external influence, the following term is added to the expression for the residual axial tension:

$$
\sigma_{e z}=\frac{P_{0}}{S_{m}(k x+1)}
$$

Where $\mathrm{P}_{0}$ is the force applied to the composite; $\mathrm{Sm}=\pi(\mathrm{Rm})^{2}$ is the microwire cross-sectional area. The theory of NFMR is presented in Refs. [5-10]. The NFMR frequency can be written as

$$
\omega(G H z) \approx \omega o\left(\frac{0.4 x}{0.4 x+1}+\frac{\sigma_{e z}}{\sigma_{0}}\right)^{1 / 2},
$$

Where

$$
\omega_{0}(G H z) \approx 1,5\left(10^{6} \lambda\right)^{1 / 2}
$$

\section{Design of Composites for Radio-Absorption Shielding}

\section{The Designs of Composites from GCAMNWs have Following Configurations}

However, for very thin GCAMNWs with negative, positive and zero magnetostriction the domain structure is far more complicated due to a complex stress distribution within the small diameter. The simplest domain structure reported so far for these GCAMNWs is obtained in $[10,12]$. As for the microwire array configuration for noise suppression experiments, 223 microwires (10 $\mu \mathrm{m}$ total diameter and length of $5 \mathrm{~mm}$ where the metal core diameter is 2 $\mu \mathrm{m}$,) are parallel aligned (Figures 1a \& 1b) in an area of $5 \times 4 \mathrm{~mm}^{2}$ as can be observed in (Figure 1c). They are supported by a thin polymer sheet ( $100 \mu \mathrm{m}$ thickness) [1-3].

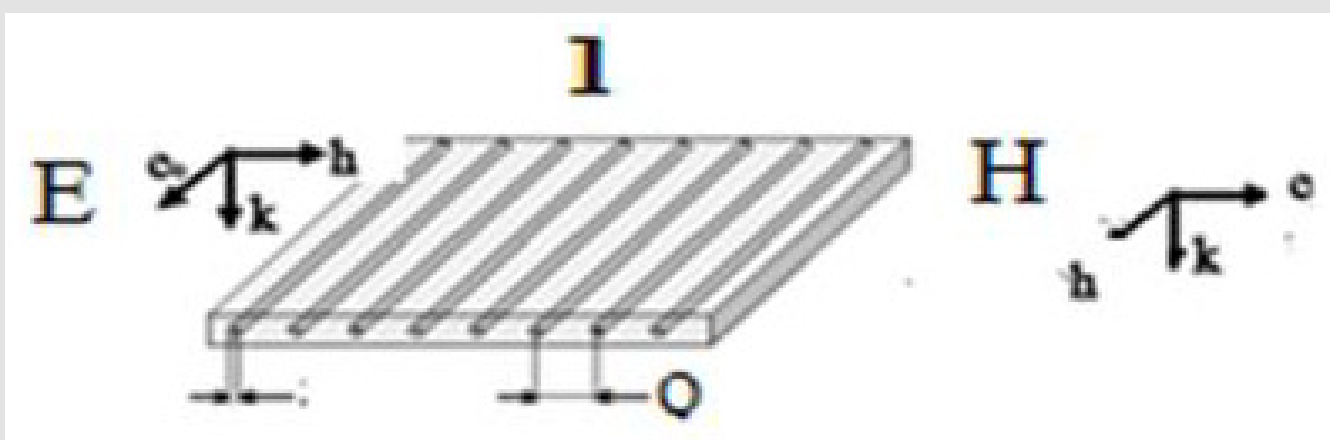

Figure 1a: Composite shielding for radio absorption protection with GCAMNWs where were maked in grating form. We can consider two types of orientation of a magnetic field: $\mathrm{E}$ and $\mathrm{H}$. 


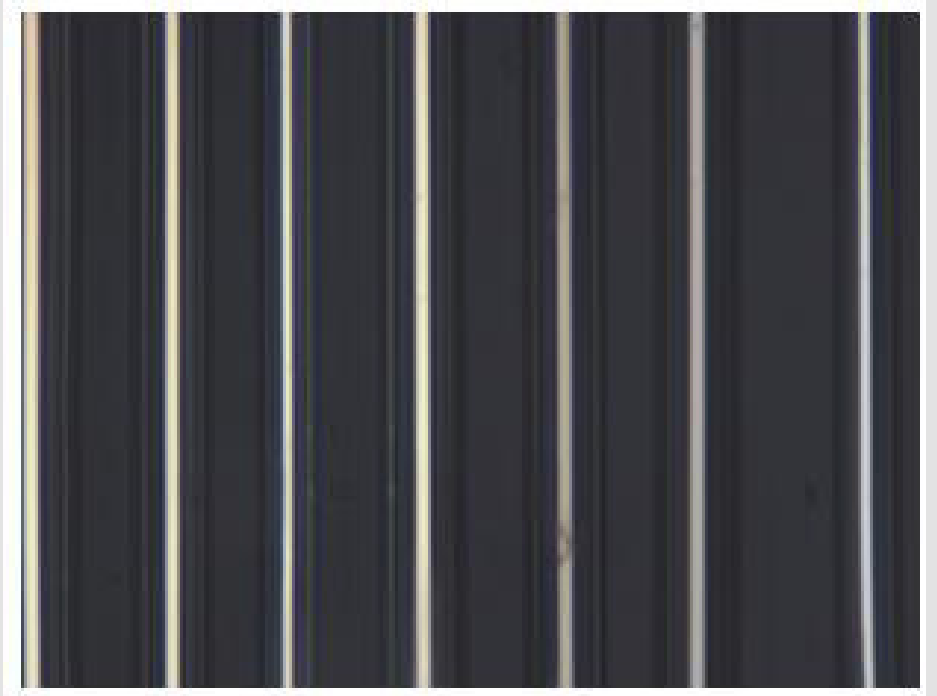

Figure 1b: Micrograph of an array of Co based microwires.

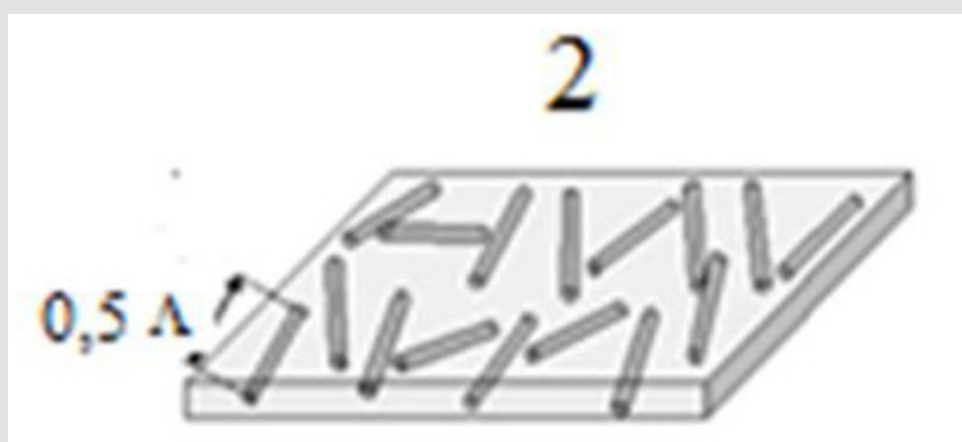

Figure 1c: Composite shielding for radio absorption protection with GCAMNWs where were made in a stochastic mixture of microwires in the polymeric matrix.

\section{Noise Absorption Experiments}

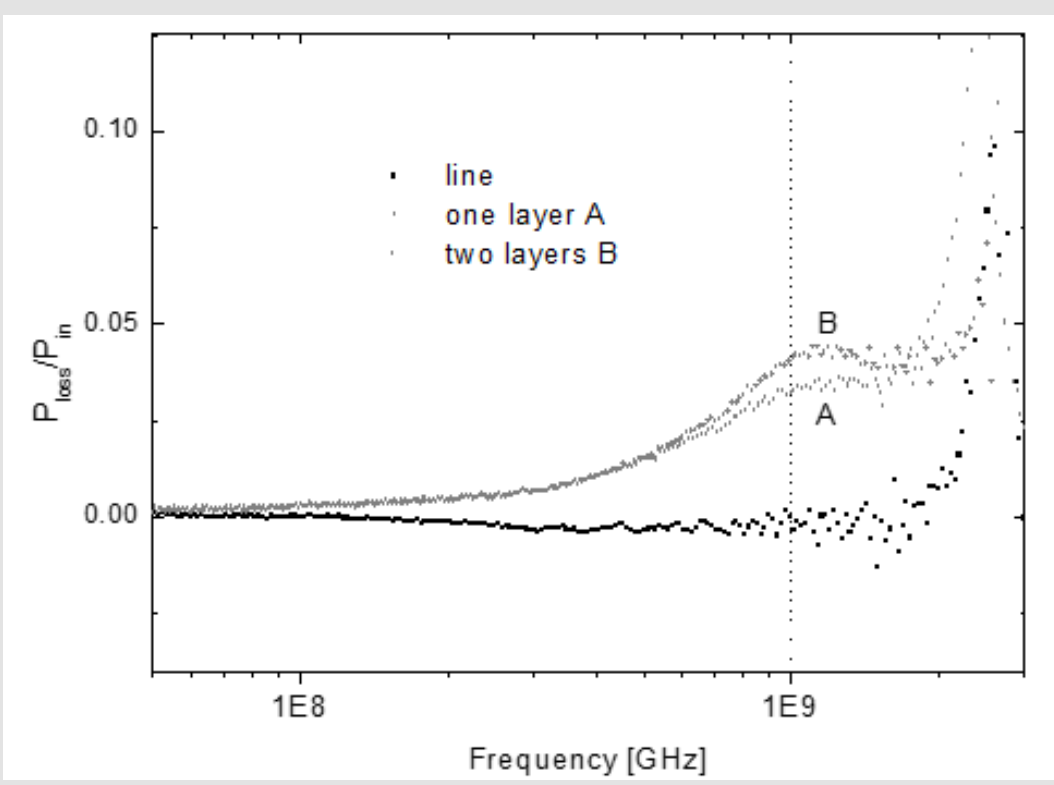

Figure 2: Power loss profile of Co based GCAMNWs shielding. 
In order to investigate the noise absorption performances a microstrip line (with $50 \Omega$ characteristic impedance) has been designed [1-3]. Similar equipment can be used to study the stress effect on the NFMR. The signal line has a width of (6-10) $\mu \mathrm{m}$ and thickness of $\mathrm{t}=35 \mu \mathrm{m}$ and is set on a 300 thick Teflon base with permittivity $\varepsilon_{r}=3.6$. The insertion losses are estimated below 0.08 at $1 \mathrm{GHz}$. The performance of the line is measured using an Agilent E8363B network analyzer from 0.1 to $20 \mathrm{GHz}$. The microwire array was placed on the top of the signal line. Samples of a single and twofold array of microwires were fabricated. The measured absorption coefficient $S_{21}$ on the microstrip line is showed in [1]. The $\mathrm{S}_{21}$ parameter at $1 \mathrm{GHz}$ decreases from the background of -0.08 $\mathrm{dB}$ to $-0.154 \mathrm{~dB}$ and $-0.188 \mathrm{~dB}$ for the single layer and twofold layer (labeled as A to B). It is worth mentioning that larger decreases are observed at around $20 \mathrm{GHz}$. This is probably due to the metallic property of the wires. The ratio of the power loss by the microwire layers to the input power calculated as $\mathrm{P}_{\text {loss }} / \mathrm{P}_{\text {in }}=1-\left(|\mathrm{S} 11|^{2}+|\mathrm{S} 21|^{2}\right)$ is shown in (Figure 2) [1]. A loss peak nearby the resonance frequency is observed around for both samples. It is noted that the twofold sample fails to be effective correspondingly. The reason is that the second layer is not close enough to interact with the near field from the microstrip line.

From the experimental results described above the following matters should be considered for future improvements. Although the amorphous wires exhibit superb frequency profiles and various advantages such as a high production rate and geometrical flexibility, the metal cores in the wires are coated with very thick glass layer resulting in a poor packing density of high permeability region in the wire array samples. If the packing density of 0.12 for the present samples were, for example 0.8 , the power loss would be about 0.3 for a single layer. This is possible by removing the glass coating. Furthermore, by designing a wire array with proper conductivity and permeability, a high-performance absorber. These issues will be treated intensively as this research work progresses in near future.

\section{Conclusion}

The high permeability features for very thin amorphous microwires for medical apparats and HF radio absorption shielding were investigated. The electromagnetic noise absorption of sheet type samples with dimensions $(5 \times 4) \mathrm{mm}^{2}$ made of 223 parallel aligned microwires was measured. The two-fold layers exhibit of $-2.5 \mathrm{~dB}$, with insertion losses below 0.2 at $1 \mathrm{GHz}$ which corresponds to the resonance of magnetization at $(1-10) \mathrm{GHz}$ [1-3]. On the other hand, we have presented simple analytical expressions for the residual and external stresses in the metallic kernel of the microwire, which clearly show their dependence on the ratio of the external radius of the microwire to the radius of the metal kernel and on the ratio of Young's modules of glass and metal. The NFMR phenomenon observed in glass-coated magnetic microwires opens up the possibility of developing new materials with a wide range of properties [6-9]. An important feature of cast microwires with an amorphous magnetic core is the dependence of the NFMR frequency from the deformation [7,8]. Therefore, this effect can be used for contactless diagnostics of deformations in distant objects (including organs of the body) reinforced by cast magnetic microwires with the stress effect. These objects are periodically scanned with floating-frequency radar to determine the deviation of the initial NFMR frequency due to potentially dangerous deformations of the monitored object.

Another principle of detecting mechanical strain is examined in $[15,16]$. This principle is based on the giant magnetoimpedance (GMI) effect. The GMI effect $[15,16]$ demands external magnetization of the sample which is not required in the NFMR method [6-9].

\section{References}

1. SA Baranov, M Yamaguchi, KL Garcia, M Vazquez (2008) Dimensional absorption high-frequency properties of the cast glass coated microwires. Surf Engin Appl Electrochem 44(6): 245-247.

2. SA Baranov, M Yamaguchi, KL Garcia, M Vazquez (2010) Application of Amorphous Microwires for Electromagnetic Shielding. Moldavian J Phys Sci 9(1): 76-82.

3. SA Baranov (2016) New Perspective for Biomedical Productions: Application of Cast Amorphous Microwire for Electromagnetic Absorption. In: Sontea V, Tiginyanu I (Eds.). $3^{\text {rd }}$ International Conference on Nanotechnologies and Biomedical Engineering 55: 259-263.

4. SA Baranov (2018) Cast amorphous magnetic microwires for medical applications. Advances in Biotechnology \& Microbiology 8(3): 50-53.

5. SA Baranov, VS Larin, AV Torcunov (2017) Technology Preparation and Properties of the Cast Glass-Coated Magnetic Microwires. Crystals 7(6): 136.

6. SA Baranov (1998) Use of a micro conductor with natural ferromagnetic resonance for radio-absorbing materials. Tech Phys Lett 24: 549-550.

7. E Adar, AM Yosher, SA Baranov (2020) Natural ferromagnetic resonance in cast microwires and its application to the safety control of infrastructures. International Journal of Engineering Research and Application 3: 118-122.

8. SA Baranov (2015) Engineering Microwave Properties of Microwires. Moldavian J Phys Sci 14(3-4): 201-214.

9. SA Baranov (2017) An engineering review about microwire. Lambert (Academic publishing) p. 1- 42.

10.SA Baranov (2011) Magnetic models of cast amorphous microwires. Surf Engin Appl Electrochem 47(4): 316-330.

11.SA Baranov (1999) Generalized conductivity and optimum energy release. Tech Phys 44: 853-855.

12.SA Baranov (2003) Magnetic properties of Co-based amorphous microwire. Journal of Magnetism and Magnetic Materials 266(3): 278281.

13. HX Peng, F Qin, MH Phan (2016) Ferromagnetic microwires composites: From sensors to microwave applications. Springer 12: 245.

14. F Qin, HX Peng (2013) Ferromagnetic microwires enabled multifunctional composite materials. Progr Mater Sci 58: 183-259.

15. MG Nematov, AM Adam, LV Panina, NA Yudanov, A Dzhumazoda, et al. (2019) Magnetic anisotropy and stress-magnetoimpedance (S-MI) in current-annealed Co-rich glass-coated microwires with positive magnetostriction. J Magn Magn Mater 474: 296-300.

16. D Makhnovskiy, A Zhukov, V Zhukova, J Gonzalez (2008) Tunable and SelfSensing Microwave Composite Materials Incorporating Ferromagnetic Microwires. Adv Sci Technol 54: 201-210. 
ISSN: 2574-1241

DOI: 10.26717/BJSTR.2021.32.005329

SA Baranov. Biomed J Sci \& Tech Res

(C) (i) This work is licensed under Creative

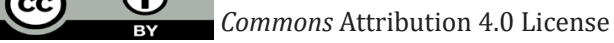

Submission Link: https://biomedres.us/submit-manuscript.php

BIOMEDICAL
RESEARCHES $\quad \begin{aligned} & \text { Assets of Publishing with us } \\ & \text { - Global archiving of articles }\end{aligned}$

